\title{
The Relationship of Spiritual Health and Mother's Forgiveness With Her Anxiety in the Labor of the Pregnant Women
}

\author{
Hanieh Dehestani $^{1}{ }^{\circledR}$, Zeinab Moshfeghy $^{2}$, Fatemeh Ghodrati ${ }^{3}$, Marzieh Akbarzadeh ${ }^{4^{*}}$
}

\begin{abstract}
Objectives: Delivery is a painful process and may impose different mental effects on the mother. The present study investigated the relationship between forgiveness and spiritual well-being on the mother's anxiety during her labor.

Materials and Methods: This is a cross-sectional and descriptive-analytic study. The research population included all the pregnant women who referred to three maternity hospitals affiliated to Shiraz University of Medical Sciences. In addition, the sample contained 200 pregnant women (both the prime and multiparous mothers) based on multiple regression analysis and 10 samples were selected for each variable of the study. The Paloutzian and Ellison Spiritual Health Scale, Pollard Forgiveness Scale in Family and Spielberger's questionnaire were used to collect the data.

Results: Based on Pearson correlation test, variables of the study demonstrated a significant relationship and the correlations between spiritual health and trait anxiety of the mothers, state anxiety, and the total score of anxiety were $\mathrm{r}=-0.329,-0.385$, and -0.363 , respectively. Further, a significant relationship was observed between mother forgiveness and mother's anxiety $(r=-0.352)$, the state anxiety $(\mathrm{r}=-0.39)$, and the anxiety variable $(\mathrm{r}=-0.377)(P=0.001)$.

Conclusions: In general, there was a significant negative relationship between spiritual health and forgiveness with the state, trait, and overall score of anxiety.

Keywords: Spiritual, Health, Forgiveness, Anxiety, Labor
\end{abstract}

\section{Introduction}

Pregnancy is a physiological phenomenon and an enjoyable period of a woman's life. In addition, childbirth is an important incident in her life (1). Each pregnant woman acts in a unique way regarding tolerating pregnancy and labor pain compared to their pregnant counterparts. Many factors affect this reaction including the mothers' training, economic status, unwanted pregnancy, abortion records, and undesirable physiological symptoms such as vaginal bleeding and fever with anxiety related to the pregnancy (2). Generally, pregnant women face anxiety, sleep disturbances, frequent waking up, nighttime sleep deprivation, and effective sleep deprivation from the 12th week of pregnancy in the first two months after the labor. These problems in the sleep pattern are due to the anxiety, or they cause anxiety (3).

Anxiety is a psychological state which occurs as a result of dealing with mental or real stressors (4). Therefore, paying attention to mother's anxiety during pregnancy is very important and the health personnel should have knowledge about the risk factors, symptoms, and interventions which reduce the mothers' anxiety. Further, anxiety reduction interventions during pregnancy can have a significant role in maternal and fetal health. Basically, hormones which are produced in response to anxiety such as catecholamine, cortisol, epinephrine, and beta-endorphins may interfere in the progression of cervical dilatation, affect the smooth muscles of the uterus, and reduce the contraction ability of the uterus and its efficacy in the process of the childbirth, which ultimately, prolong the delivery, increase pain, and cause anxiety (5).

Furthermore, the mother's anxiety and unpleasant mood during her pregnancy increase the maternal cortisol and the adrenal secretion in the fetus, which directly influences the mood of the fetus $(3,6)$.

Babies of those mothers who have severe anxiety during their pregnancy are restless, cry more and demonstrate further behavioral changes compared to those of nonanxiety mothers $(7,8)$. One study $(6)$ found that maternal anxiety affects the time and variability of the fetus's heart rate and mimics long-lasting and rising patterns (tachycardia).

Moreover, tension and negative experiences of mothers during their pregnancy have a negative impact on physical growth, motor-behavioral development, and the 
psychological dimension of the newborn (9). According to the estimation of the World Health Organization, more than $80 \%$ of the women with low-risk pregnancies experience some degree of anxiety during pregnancy. Additionally, one woman among every three to five pregnant women has anxiety problems in developing countries while this rate is one woman in every 10 pregnant women in developed countries (10).

Accordingly, several methods were reported for reducing the rate of anxiety in various studies. For example, yoga, slowly moving the body, and massage can help reduce maternal anxiety while these exercises improve pregnancy outcomes (11). In addition, based on the findings of one study the growth restriction of the fetus of depressed and distressed women who received massage therapy during pregnancy and labor was $75 \%$ less than that of women without massage therapy. Further, the rate of birth with low weight reduced by $80 \%$. Finally, the anxiety and depression scores of pregnant women decreased (12).

Given the impact of pregnant women' anxiety problems on the growth and health of the fetus, the present study aimed to determine the dimensions of mothers' spiritual well-being and their forgiveness trait and its relationship with labor anxiety. In $94 \%$ of the studies, it was observed that patients equally emphasize both their spiritual and physical well-being. A study regarding the family physicians found that $96 \%$ of the respondents believed that mental health was an important factor in health; however, the spiritual health of the patients was frequently neglected (13).

Furthermore, the results of various studies indicated that patients with physical injuries and suffering have a tendency toward religious beliefs since religion helps them tolerate the suffering and pain arising from their disease. Similarly, Al Zaben et al (14) represented that religious beliefs enable the patients to achieve widespread social support and motivate them to accept treating the disease and coping with the related stress.

For instance, faithful patients have high expectations for religious experts' intervention during suffering from the illness and believe that their cancer can be treated by the prayer of the others. Moreover, meeting members of the mosque and their support and encouragement have an effect on the patients' health (14).

Based on the results of another study, relationship with God and praying was considered a great deal of hope reported by the patients. Additionally, other spiritual sources, family members, health care workers, and the medical team played an important role in their treatment. Relationship with God and support of their families was found to give more hope to the patients and increase their recovery (15).

In addition, the ability to forgive is regarded as one of the most essential and influential variables on the mental health of the individuals, which was greatly emphasized. The main religions of the world including
Islam, Christianity, and Judaism encouraged forgiveness. For example, in Islam, there are many verses in the Quran about forgiving those who bothered you, and even recommending that: "You should forgive people who bothered you and behave well toward such people" (16).

Tse and Yip found a significantly positive relationship between forgiveness and psychological well-being and interpersonal adjustment. Therefore, these two variables can be improved in individuals and they may increase the physical dimensions and psychological well-being of the individuals throughout their lives (17).

Since the obstetricians are involved in reproduction of humans, then obstetrics is always an up-to-date issue and appropriate to the present situation. The goal of this specialty is to promote health and increase the wellbeing of the pregnant woman and her fetus by highquality prenatal cares. The importance of the obstetrics is permanently increasing given that the maternal and neonatal outcomes are used as one of the indicators of the quality of life and health of human societies. Further, the midwife is the one who has the most chance to associate with the pregnant woman during her pregnancy, therefore, highlighting the importance of her role in counseling, training, and supporting the pregnant women is essential. Considering the lack of sufficient studies in this field, the present research was conducted to examine and improve the quality of the mother and baby's physical and mental health. Accordingly, the study investigated the relationship between spiritual health and forgiveness of the mother with maternal anxiety during her labor.

\section{Materials and Methods}

Participants

Given the statistical consultation, the sample size included 200 based on multiple regression analysis and 10 samples were selected for each variable of the current study. Totally, 100 subjects were primigravida while 100 of them were multigravida and were selected using a simple sampling method. Furthermore, those women who had the criteria were included in the study. The inclusion criteria were lack of high-risk pregnancy (i.e., twin pregnancies, problems with the placenta and fetus, the pregnancy age of 37-42 weeks, absence of internal disease, mother and fetus surgery, fetal distress in the beginning, mental illness, and lack of using psychoactive drugs including imipramine, and the like) and being in the active phase of the labor (at least $4 \mathrm{~cm}$ dilatation). Moreover, the exclusion criterion included the mothers who disagree to continue participating in the project in which case, they could discontinue the study.

\section{Design}

This study was of the cross-sectional descriptive-analytic type and its population encompassed all the pregnant women who referred to three maternity hospitals affiliated to Shiraz University of Medical Sciences. 
Tasks and Procedure

Totally, 4 instruments were used for data collection.

\section{Demographic Questionnaire}

The first part of this questionnaire contained personal and general information and the second part included medical information and pregnancy records.

\section{Paloutzian and Ellison Spiritual Health Scale}

This scale included twenty questions on religious health $(n=10)$ and measuring the health of a person $(n=10)$. The spiritual health score of these two subgroups was between 20 and 120. The questions were answered on a 6-point Likert-type scale ranging from "Completely agree" to "Completely disagree". Finally, the spiritual health of the people was divided into 3 low (20-40), medium (41-99), and high (100-120) groups. Paloutzian and Ellison reported a Cronbach's Alpha coefficient of 0.91, 0.91, and 0.93 for the questionnaire. The Cronbach's alpha coefficient for this questionnaire has been estimated by Heydarzadegan in Iran, as quoted from Elaheh Bakhshiyan, to be 0.82 $(18,19)$.

\section{Forgiveness Scale}

This scale was designed and developed by Pollard et al to determine the level of forgiveness in families and dimensions of forgiveness. The main form of the scale contained 40 phrases which referred to the state of anger and annoyance, along with forgiveness among the family members. Each phrase included options " $4=$ almost always true", " 3 = often true", " 2 = seldom true", and " $1=$ never true". High and low levels indicated the presence and lack of forgiveness in the family, respectively. Bahari and Seif studied the norms to estimate the psychometric properties related to the measuring scale of the community's forgiveness in the created family of Pollard et al (20). The Cronbach's Alpha coefficient of the forgiveness scale in the family was 84.5 , indicating that the questionnaire enjoyed a satisfactory level of reliability (21).

\section{Spielberger State-Trait Anxiety Inventory}

Anxiety Scale was used to measure anxiety including 40 questions and 80 grades. This test encompassed 40 questions evaluating the trait anxiety (20 items) and state anxiety (20 items). The total scores of both trait and state anxiety scales were in the range of 20-80. The scores related to these items were classified into 4 groups including 0-19 (natural anxiety), 20-40 (mild anxiety), 41-60 (moderate anxiety), and 61-80 (severe anxiety) and higher scores indicated a higher rate of anxiety. In the case of Spielberger anxiety test, Mahram et al standardized this questionnaire in Iran and calculated its reliability as 0.91 by the Cronbach alpha. The reliability obtained by Mahram et al was the basis for the present study (22).

\section{Results}

Among the 200 individuals in the sample group, 17 (8.5\%) cases were less than 20 years. Additionally, the highest $(n=61,30.5 \%)$ and lowest $(n=7,3.5 \%)$ frequencies belonged to those who were $30-26$ and over 40 years old, respectively. In addition, 154 (79\%) of whom have less than a diploma, 14 (7\%) have a diploma, and 32 (14\%) have university degrees. Further, based on the results, the number of women suffering from different levels of anxiety (i.e., mild, moderate, relatively severe, and severe) included 111 (55.5\%), 42 (21\%), 43 (21.5\%), and 4 (2\%) women, respectively. Furthermore, the mean and standard deviation (SD) of anxiety was $36.15 \pm 15.37$ (Table 1 ).

Based on results of Pearson correlation test, the correlation between spiritual health and maternal trait and state anxiety and the total score of anxiety was obtained $-0.329,-0.385$, and -0.363 , respectively, with a significance level of 0.0001 which was 0.05 lower than the assumed error in the study. Therefore, there was a significant negative relationship between spiritual health and state anxiety, and between the trait anxiety and the mother's anxiety. In other words, higher spiritual health, red to the lower level of the state, trait, and mother's anxiety (Tables 2 and 3). Moreover, Pearson correlation tests revealed that the correlation between mother forgiveness and mother's anxiety, the state anxiety, and the anxiety was equal to $-0.352,-0.39$, and -0.377 , respectively, with a significance level of 0.0001 , indicating 0.05 lower than the assumed error in the research. Accordingly, a significant negative relationship was detected between the mother's forgiveness and state anxiety, as well as the trait of anxiety and the mother's anxiety. Therefore, the higher level of the mother's forgiveness indicated a lower level of state anxiety, trait anxiety, and mother's anxiety (Tables 4 and 5).

Table 1. Frequency and Percent Frequency of Anxiety Variable in the Sample Group

\begin{tabular}{|c|c|c|c|c|c|c|c|c|}
\hline & \multirow{2}{*}{ No. } & \multirow{2}{*}{$\%$} & \multicolumn{2}{|c|}{ Total Anxiety } & \multicolumn{2}{|c|}{ Situational Anxiety } & \multicolumn{2}{|c|}{ Personality Anxiety } \\
\hline & & & Mean & SD & Mean & SD & Mean & SD \\
\hline Normal & 0 & 0 & & & & & & \\
\hline Mild & 111 & 55.5 & & & & & & \\
\hline Medium & 42 & 21 & 36.15 & 15.37 & 18.07 & 7.60 & 18.08 & 8.01 \\
\hline Fairly intense & 43 & 21.5 & & & & & & \\
\hline Intense & 4 & 2 & & & & & & \\
\hline Total & 200 & 100 & & & & & & \\
\hline
\end{tabular}


Table 2. Frequency Values for the Differentiation of Spiritual Health Variables

\begin{tabular}{llccc}
\hline & & \multicolumn{3}{c}{ Spiritual Health } \\
\cline { 3 - 5 } & & Medium & Top & Total \\
\hline \multirow{3}{*}{ Mother's } & Medium & 20 & 91 & 111 \\
anxiety & Fairly intense & 24 & 19 & 42 \\
& Intense & 1 & 3 & 4 \\
Total & & 63 & 137 & 200 \\
\hline
\end{tabular}

Table 3. Correlations of Spiritual Health With Mother's Anxiety

\begin{tabular}{lccc}
\hline & Correlation Coefficient & Number & $\boldsymbol{P}$ Value \\
\hline Situational anxiety & -0.329 & 200 & 0.0001 \\
Personality anxiety & -0.385 & 200 & 0.0001 \\
Total anxiety & -0.363 & 200 & 0.0001 \\
\hline
\end{tabular}

Table 4. Frequent Amounts of Maternal Forgiveness and Anxiety

\begin{tabular}{llccc}
\hline & & \multicolumn{3}{c}{ Mother Forgiveness } \\
\cline { 3 - 5 } & & Medium & Top & Total \\
\hline \multirow{3}{*}{ Mother's } & Mild & 17 & 94 & 111 \\
anxiety & Fairly intense & 18 & 25 & 43 \\
& Intense & 1 & 3 & 4 \\
Total & & 50 & 150 & 200 \\
\hline
\end{tabular}

Table 5. Correlation Values of Maternal Forgiveness and Anxiety

\begin{tabular}{lccc}
\hline & Correlation Coefficient & Number & $\boldsymbol{P}$ Value \\
\hline Situational anxiety & -0.352 & 200 & 0.0001 \\
Personality anxiety & -0.39 & 200 & 0.0001 \\
Total anxiety & -0.377 & 200 & 0.0001 \\
\hline
\end{tabular}

\section{Discussion}

Having the trait of forgiveness and paying attention to empathic perspectives can reduce the level of psychological stress (23). The results showed that $55.5 \%$ of the population of the study had a mild anxiety, $21 \%$ suffered from moderate anxiety, $21.5 \%$ had a relatively severe anxiety and $2 \%$ had intensive. Additionally, a significant relationship was observed between mental health and the mother's anxiety (i.e., situational, personality, and general anxiety scores). To evaluate the anxiety of pregnant mothers in their third trimester of pregnancy and its related factors, a study was performed by Sadeghi et al including 75 pregnant women who referred to Bandar Abbas hospitals. The results of the study demonstrated that $42.6 \%$ of the women had clearly moderate and severe anxiety while $57.3 \%$ of them suffered from mild anxiety. In addition, $45.3 \%$ of these pregnant women had hidden moderate and severe anxiety whereas $54.6 \%$ of them suffered from mild anxiety. This is in line with the results of the present study, indicating that the majority of pregnant women experienced a mild level of anxiety (24).
Further, Jabbari et al investigated the effect of Holly Quran vocalization with and without translation on the level of stress, anxiety, and depression during pregnancy among Iranian pregnant women and found that the religious beliefs reduced the anxiety (25).

Based on the findings of the current study, continuous training programs should be offered for pregnant women by health personnel based on the needs of these women and their access to pregnancy services in order to reduce their level of anxiety. Furthermore, establishing special clinics for pregnant women can be an effective step for training programs during the pregnancy, improving mental status and pregnancy problems, and creating a more comfortable feeling in pregnant women.

Moreover, using different training programs (26-29) and training regarding religious beliefs and morality is frequently considered a constructive coping strategy for improving the mental health of the individuals. Interventional research indicated that training, awareness, and spiritual attitude can reduce anxiety and depression while it can promote the mental health of the mother and fetus, as well as non-pregnant women (30-33).

The women in each of the above-mentioned groups were in a special situation which created anxiety for each individual of the group; it was proved that in all the studies similar to the present study, people can reduce their anxiety by increasing their spiritual health under any conditions. Additionally, a significant negative relationship was found between anxiety and spiritual well-being of the mothers.

In the current study, there was a significant negative relationship between mother's forgiveness and trait anxiety, as well as mother's anxiety and state anxiety. Therefore, the higher level of the mother' forgiveness decreased the level of trait anxiety and the mother's anxiety (34). Over the past 2 decades, the issue related to the trait of forgiving and society's health has attracted a lot of attention and its role in promoting maternal health has increasingly recognized. Although forgiveness is confirmed to be useful, further studies are needed in this respect.

In a study conducted by Lavafpour Nouri et al, the effect of the psychological-training protocol of the forgiveness was investigated on depression, anxiety, and stress among adolescents. The findings represented that forgiveness training can reduce the symptoms of depression, anxiety, and stress among the students. Although contrary to the present study, this study was interventional, and most importantly, it was not related to pregnant women, similar to the present study, an increase was observed in forgiveness score while the level of anxiety decreased (35).

In another study, Zhang et al reported that forgiveness intervention was effective in dealing with interpersonal problems among the students whose romantic relationship was damaged. In addition, they found a significant improvement in anxiety, depression, and well-being while only the forgiveness group demonstrated a meaningful improvement in forgiveness. Further, the effectiveness of 
treatment for the forgiveness group was longer compared to the control group (36).

Furthermore, Yazla et al investigated 100 diabetic patients with various socio-demographic characteristics, emotional problems, and glycemic control, as well as the relationship between religious beliefs and forgiveness. They emphasized that forgiveness by the patient or others has a relationship with emotional problems and can reduce the level of stress. Finally, it was revealed that forgiveness increases the quality of life of diabetic patients (37).

Considering the fact that few studies were performed regarding the forgiveness, especially in the process of pregnancy, the above studies demonstrated that forgiveness can have a positive effect on the reduction of anxiety in the lives of individuals.

Anxiety is common during pregnancy and thus can have a negative effect on the health of the mother, and fetus, as well as the newborn baby. Therefore, attention to anxiety in pregnancy is of great importance. Prenatal visits were considered a suitable opportunity to train pregnant mothers and training interventions were found to reduce the rate of anxiety $(38,39)$.

\section{Conclusions}

Based on the results, more than half of the mothers had mild anxiety while $21.5 \%$ of them suffered from relatively severe anxiety. Moreover, there was a significant negative relationship between the forgiveness-spiritual health and mother's state anxiety, as well as between trait anxiety and a total score of anxiety. Therefore, developing programs concerning spiritual training and strengthening the trait of forgiveness for evaluating the mental health of mothers in prenatal cares seem to be logical.

\section{Conflict of Interests}

Authors declare that they have no conflict of interests.

\section{Ethical Issues}

This research project was approved by the local Ethics Committee of Shiraz University of Medical Sciences (ethical code: IR.sums.REC.1395.S717) and written informed consents were obtained from all the participants. Additionally, the research proposal No. 12362 was financially supported by the Research Vice-chancellor of Shiraz University of Medical Sciences.

\section{Financial Support}

This article is a part of the thesis (No. 12362) submitted by Hanieh Dehestani. The study was supported by Research Improvement Center of Shiraz University of Medical Sciences.

\section{Acknowledgments}

Hereby, the authors would like to thank the deans of the prenatal clinics of Hafez, Zeinabiyeh, and Soushtari Hospitals affiliated to Shiraz University of Medical
Sciences. Special thanks go to Shiraz University of Medical Sciences and Center for Development of Clinical Research of Nemazee Hospital, as well as Dr. Nasrin Shokrpour for editorial assistance. In addition, researchers would appreciate Research and Technology Department, as well as Research Improvement Center of Shiraz University of Medical Sciences for financial support.

\section{References}

1. Cunningham FG, Leveno KJ, Bloom SL, Hauth JC. Williams Obstetrics. 23rd ed. Michigan, USA: McGrawHill; 2010:673-679.

2. Ding X, Mao L, Ge X, et al. [Course and risk factors of maternal pregnancy-related anxiety across pregnancy in Ma’ anshan city]. Wei Sheng Yan Jiu. 2015;44(3):371-375.

3. Punamaki RL, Repokari L, Vilska S, et al. Maternal mental health and medical predictors of infant developmental and health problems from pregnancy to one year: does former infertility matter? Infant Behav Dev. 2006;29(2):230-242. doi:10.1016/j.infbeh.2005.12.001

4. Faisal-Cury A, Menezes P, Araya R, Zugaib M. Common mental disorders during pregnancy: prevalence and associated factors among low-income women in Sao Paulo, Brazil: depression and anxiety during pregnancy. Arch Womens Ment Health. 2009;12(5):335-343. doi:10.1007/ s00737-009-0081-6

5. Hall WA, Hauck YL, Carty EM, Hutton EK, Fenwick J, Stoll K. Childbirth fear, anxiety, fatigue, and sleep deprivation in pregnant women. J Obstet Gynecol Neonatal Nurs. 2009;38(5):567-576. doi:10.1111/j.1552-6909.2009.01054.x

6. Akbarzadeh M, Dokuhaki A, Joker A, Pishva N, Zare N. Teaching attachment behaviors to pregnant women: a randomized controlled trial of effects on infant mental health from birth to the age of three months. Ann Saudi Med. 2016;36(3):175-183. doi:10.5144/0256-4947.2016.175

7. Sanders KA, Bruce NW. Psychosocial stress and treatment outcome following assisted reproductive technology. Hum Reprod. 1999;14(6):1656-1662.

8. Sternfeld B, Quesenberry CP Jr, Eskenazi B, Newman LA. Exercise during pregnancy and pregnancy outcome. Med Sci Sports Exerc. 1995;27(5):634-640.

9. Dokuhaki A, Akbarzadeh M, Pishva N, Zare N. A study of the effect of training pregnant women about attachment skills on infants' motor development indices at birth to four months. Fam Med Prim Care Rev. 2017;19(2):114-122. doi:10.5114/fmpcr.2017.67864

10. World Health Organization (WHO). Maternal mental health and child health and development in low and middle income countries. Geneva: WHO; 2008.

11. Saisto T, Toivanen R, Salmela-Aro K, Halmesmaki E. Therapeutic group psychoeducation and relaxation in treating fear of childbirth. Acta Obstet Gynecol Scand. 2006;85(11):1315-1319. doi:10.1080/00016340600756920

12. Bastani F, Hidarnia A, Kazemnejad A, Vafaei M, Kashanian M. A randomized controlled trial of the effects of applied relaxation training on reducing anxiety and perceived stress in pregnant women. J Midwifery Womens Health. 2005;50(4):e36-40. doi:10.1016/j.jmwh.2004.11.008

13. Mueller PS, Plevak DJ, Rummans TA. Religious involvement, spirituality, and medicine: implications for clinical practice. Mayo Clin Proc. 2001;76(12):1225-1235. 
doi:10.4065/76.12.1225

14. Al Zaben F, Khalifa DA, Sehlo MG, et al. Religious involvement and health in dialysis patients in Saudi Arabia. J Relig Health. 2015;54(2):713-730. doi:10.1007/s10943014-9962-8

15. Afrooz R, Rahmani A, Zamanzadeh V, et al. The nature of hope among Iranian cancer patients. Asian Pac J Cancer Prev. 2014;15(21):9307-9312.

16. Elahi-Ghomsheie M. Holy Quran with Persian translation, explanation and searching the explanation. Tehran: Marvi Publication; 1993. [Persian].

17. Tse WS, Yip THJ. Relationship among dispositional forgiveness of others, interpersonal adjustment and psychological well-being: Implication for interpersonal theory of depression. Pers Individ Dif. 2009;46(3):365-368. doi:10.1016/j.paid.2008.11.001

18. Thoresen CE, Harris AH, Luskin F. Forgiveness and health: An unanswered question. In: McCullough ME, Pargament KI, Thoresen CE, eds. Forgiveness: Theory, research, and practice. New York: Guilford Press; 2001:254-280.

19. Heydarzadegan A, Koochakzaei M. Study of the relationship between spiritual well-being and self-efficacy of students of faculty of engineering and psychology and educational sciences. Iranian Journal of Engineering Education. 2015;17(65):79-93. doi:10.22047/ijee.2015.8012

20. Pollard MW, Anderson RA, Anderson WT, Jennings G. The development of a family forgiveness scale. J Fam Ther. 1998;20(1):95-109. doi:10.1111/1467-6427.00070

21. Bahari F, Seif S. Forgiveness: Introducing a therapeutic model in marital counseling \& family therapy. Counseling Research and Development. 2001;2(7-8):49-66.

22. Mahram B. Standardization of Spielberger's test anxiety inventory in Mashhad [Thesis]. Tehran: Allameh Tabatabaei University; 1994:76. [Persian].

23. vanOyen Witvliet C, Ludwig TE, Vander Laan KL. Granting forgiveness or harboring grudges: implications for emotion, physiology, and health. Psychol Sci. 2001;12(2):117-123. doi:10.1111/1467-9280.00320

24. Sadeghi N, Azizi S, Molaeinezhad M. Anxiety status in pregnant mothers at third trimester of pregnancy and its related factors in referrals to Bandar Abbas Hospitals in 2012. The Iranian Journal of Obstetrics, Gynecology and Infertility. 2014;17(122):8-15. doi:10.22038/ijogi.2014.3574

25. Jabbari B, Mirghafourvand M, Sehhatie F, MohammadAlizadeh-Charandabi S. The effect of holly Quran voice with and without translation on stress, anxiety and depression during pregnancy: a randomized controlled trial. J Relig Health. 2017. doi:10.1007/s10943-017-0417-x

26. Toosi M, Akbarzadeh M, Sharif F, Zare N. The Reduction of Anxiety and Improved Maternal Attachment to Fetuses and Neonates by Relaxation Training in Primigravida Women. Women's Health Bull. 2014;1(1):e18968. doi:10.17795/whb18968

27. Moradi Z, Akbarzadeh M, Moradi P, Toosi M, Hadianfard
MJ. The Effect of Acupressure at GB-21 and SP-6 Acupoints on Anxiety Level and Maternal-Fetal Attachment in Primiparous Women: a Randomized Controlled Clinical Trial. Nurs Midwifery Stud. 2014;3(3):e19948.

28. Akbarzadeh M, Rafiee B, Asadi N, Zare N. Comparative Effect of Attachment and Relaxation Training on Perception of Fetal Movement and Mother's Anxiety in Primiparous Women: A Randomized Controlled Study. Trends Med Res. 2016;11(2):62-68. doi:10.3923/tmr.2016.62.68

29. Toosi M, Akbarzadeh M, Ghaemi Z. The Effect of Relaxation on Mother's Anxiety and Maternal-Fetal Attachment in Primiparous IVF Mothers. J Natl Med Assoc. 2017;109(3):164-171. doi:10.1016/j.jnma.2017.03.002

30. Mokhtaryan T, Yazdanpanahi Z, Akbarzadeh M, Amooee $\mathrm{S}$, Zare N. The impact of Islamic religious education on anxiety level in primipara mothers. J Family Med Prim Care. 2016;5(2):331-337. doi:10.4103/2249-4863.192314

31. Akbarzadeh M, Mokhtaryan T, Amooee S, Moshfeghy Z, Zare N. Investigation of the effect of religious doctrines on religious knowledge and attitude and postpartum blues in primiparous women. Iran J Nurs Midwifery Res. 2015;20(5):570-576. doi:10.4103/1735-9066.164586

32. Moritz S, Kelly MT, Xu TJ, Toews J, Rickhi B. A spirituality teaching program for depression: qualitative findings on cognitive and emotional change. Complement Ther Med. 2011;19(4):201-207. doi:10.1016/j.ctim.2011.05.006

33. Ghodrati F, Akbarzadeh M. A Review of the Importance of Maternal-fetal Attachment According to the Islamic Recommendations. Journal of Midwifery and Reproductive Health. 2018;6(1):1193-1200. doi:10.22038/jmrh.2017.9616

34. Gangdev P. Forgiveness: A note for psychiatrists. Indian J Psychiatry. 2009;51(2):153-156. doi:10.4103/00195545.49459

35. Lavafpour Nouri F, Zahrakar K, Omara S, Azarhoush Fatideh Z, Pourshojaei A, Azarhoush Fatideh N. Effect of Psychoeducational Forgiveness Program on Symptoms of Depression, Anxiety, and Stress in Adolescents. Journal of Mazandaran University of Medical Sciences. 2015;25(123):191-196.

36. Zhang T, Fu H, Wan Y. The application of group forgiveness intervention for courtship-hurt college students: a Chinese perspective. Int J Group Psychother. 2014;64(3):298-320. doi:10.1521/ijgp.2014.64.3.298

37. Yazla E, Karadere ME, Kucukler FK, et al. The Effect of Religious Belief and Forgiveness on Coping with Diabetes. J Relig Health. 2018;57(3):1010-1019. doi:10.1007/s10943017-0504-z

38. Toosi M, Akbarzadeh M, Zare N, Sharif F. Effect of attachment training on anxiety and attachment behaviors of first-time mothers. Hayat. 2011;17(3):69-79.

39. DiPietro JA, Costigan KA, Nelson P, Gurewitsch ED, Laudenslager ML. Fetal responses to induced maternal relaxation during pregnancy. Biol Psychol. 2008;77(1):1119. doi:10.1016/j.biopsycho.2007.08.008

(c) 2019 The Author (s); This is an open-access article distributed under the terms of the Creative Commons Attribution License (http://creativecommons.org/licenses/by/4.0), which permits unrestricted use, distribution, and reproduction in any medium, provided the original work is properly cited. 